\title{
Model-based noise estimation for fringe projection systems
}

\author{
Marc Fischer, Marcus Petz, Rainer Tutsch \\ Technische Universität Braunschweig, Institut für Produktionsmesstechnik \\ Schleinitzstraße 20, 38106 Braunschweig, GERMANY
}

\begin{abstract}
For full-field optical 3D measurement systems, camera noise is the dominant uncertainty factor when optically cooperative surfaces are measured in a stable and controlled environment. In industrial applications repeated measurements are seldom executed for this kind of measurement systems. This leads to statistically suboptimal results in subsequent evaluation steps as the important information about the quality of individual measurement points is lost. In this work it will be shown that this information can be recovered for phase measuring optical systems with a model-based noise prediction. The capability of this approach will be demonstrated exemplarily for a fringe projection system and it will be shown that this method is indeed able to generate an individual estimate for the spatial stochastic deviations resulting from image sensor noise for each measurement point.
\end{abstract}

Keywords: fringe projection, camera model, EMVA 1288, phase noise, noise estimation

\section{Introduction}

For full-field optical 3D measurement systems, like fringe projection systems, the achievable accuracy mainly depends on the environment conditions and the properties of the measurement object. In a production-related environment systematic deviations caused for example by temperature fluctuations or vibrations are the dominant factors. In contrast, a wellcontrolled environment leads to a strong influence of the surface properties of the object under test. While the deviations are correlated to the micro-topography in the case of noncooperative surfaces, stochastic deviations caused by camera noise come to the fore in the case of cooperative surfaces. Depending on the local lighting conditions these can differ in up to an order of magnitude for different measurement points on the object.

In industrial applications of full-field optical 3D measurement systems usually the measurements are not repeated for estimating repeatability. The deviations of the measurement process may be characterized by measurements of spherical and planar artifacts in the context of acceptance test and re-verifications according to VDI 2634 [1]. It has to be pointed out however that this is not to be confused with a measurement uncertainty as it is only a method to ensure that the system is working in conformity with the specifications.

The experimental determination of a taskspecific measurement uncertainty, for example according to VDA5 [2], is only worth the effort in the case of a small measurement object portfolio and large lot sizes. In addition this method characterizes the whole measurement process only in a general way.

The missing assessment of the quality of each individual measurement point leads to statistically suboptimal results in subsequent evaluation steps as for example this information is not available as a weighting factor for the matching of geometric primitives. Because for the usecase outlined above, i.e. the measurement of cooperative surfaces in a well-controlled environment, the geometric deviations of the measured points are dominated by camera noise, they can be estimated directly from the measurement data if the noise characteristic of the measurement process is modelled. This approach will be described in the following sections and is a first important step towards a task-related uncertainty for this kind of measurement systems.

\section{Optical measurement systems with structured illumination}

Optical measurement systems that use intensity patterns to spatially encode an object are referred to as "structured illumination" techniques. Among others, a widely applied coding approach is based on multiple phase-shifted sinusoidal fringe patterns. In this case the actual coding takes place in the time domain, as the phase-shifted patterns are usually recorded sequentially. The recorded intensity $I_{i}$ for each pixel in the $i$-th phase-shifted image can be written as: 
$I_{i}=I_{\text {sat }} \beta\left[1+\gamma \cos \left(\varphi+\psi_{i}\right)\right] ; \quad i=1, \ldots, M$

where $I_{\text {sat }}$ is the saturation intensity of the pixel, $\beta$ the relative unmodulated intensity (illumination) and $\gamma$ the relative fringe contrast (visibility), $\varphi$ the unknown phase angle, $\psi_{i}$ the applied phase-shift and $M$ the number of phase-shifted images. The parameters $\beta$ and $\gamma$ describe the local lighting conditions for a given pixel independent of the camera. Both are in the range of [0...1] with an additional constraint for the maximum of $\beta$ for a given $\gamma$ because $I_{i}$ should always be smaller than $I_{\text {sat }}$. The effect of $\beta$ and $\gamma$ on the recorded images can be visualized by means of a fringe pattern with locally varying visibility and illumination as demonstrated in fig. 1. As can be seen the optimal fringe contrast can be achieved for $\beta=0.5$ and $\gamma=1$.

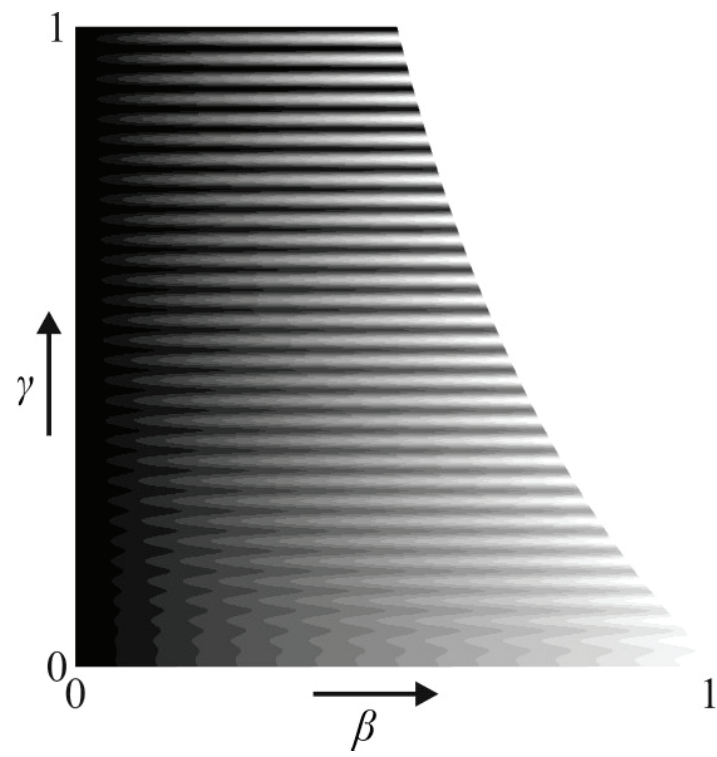

Fig. 1: Fringe pattern with locally varying visibility $\gamma$ and illumination $\beta$. In the right part of the diagram, points that do not meet the constraint for the maximum illumination (clipping) are painted white.

With the recorded intensities $I_{i}$ according to eq. (1) the corresponding unknown phase value can be calculated for each pixel by means of phase-shift algorithms. A class of widely applied algorithms is based on evenly spaced phase-shifts over one period ("symmetric $M$ step algorithms"), with the famous 4-step algorithm for $M=4$ :

$$
\tan \varphi=\frac{I_{3}-I_{1}}{I_{4}-I_{2}} ; \quad \psi_{i}=\left(\frac{\pi}{2} ; \pi ; \frac{3 \pi}{2} ; 2 \pi\right)
$$

\section{Modelling and prediction of phase noise}

For symmetric $M$-step algorithms a simple relation between the absolute unmodulated intensity $I^{\prime}$ the intensity noise of the image sensor $\sigma_{I}$ and the phase-noise $\sigma_{\varphi}$ can be found according to [3] as:

$$
\sigma_{\varphi}=\sqrt{\frac{2}{M}} \frac{1}{\gamma} \frac{\sigma_{I}}{I^{\prime}}=\sqrt{\frac{2}{M}} \frac{1}{\gamma} \frac{1}{S N R}
$$

Although this expression is based on the assumption of signal-independent Gaussian noise $\left(\sigma_{I}=\right.$ const. $)$, where the signal-to-noise ratio $(S N R)$ would increase linearly with the unmodulated intensity $I^{\prime}$, in [4] it has been shown that it is also valid for a more complex expression of $S N R$ resulting from the advanced linear camera model of the guideline EMVA 1288 [5]. Furthermore it has been demonstrated in [4], that eq. (3) can be developed into a prediction method able to estimate the phase noise directly from the measured intensities. The basic physical model of signal generation in a camera pixel is shown in fig. 2 (a), the resulting mathematical model in fig. 2 (b).

(a)

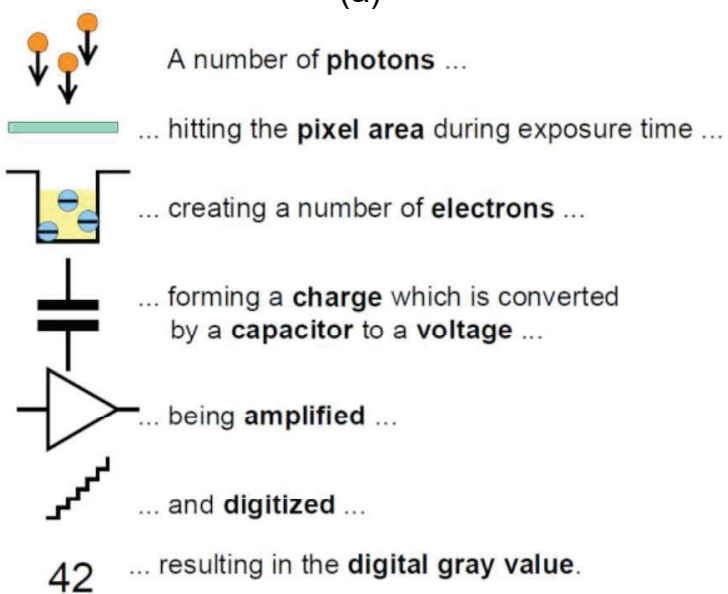

(b)

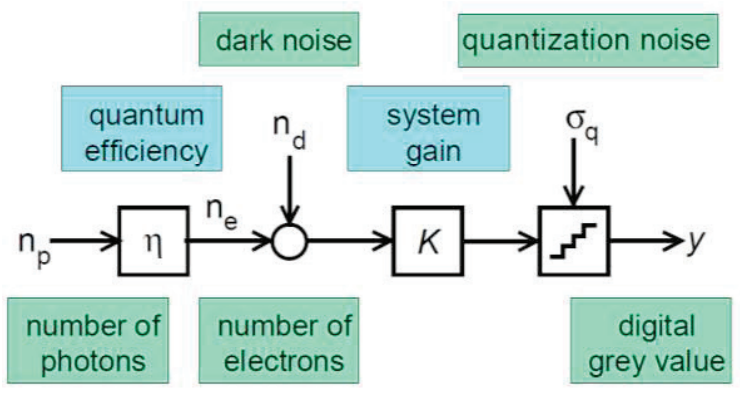

Fig. 2: Physical model of signal generation in a CCD-camera pixel according to EMVA 1288 (a) and corresponding mathematical camera model (b). [5] 
It can be seen that the fundamental process is a conversion, first from number of photons $n_{p}$ to number of electrons $n_{e}$ with the total quantum efficiency $\eta$ as the conversion factor and second to digital gray values $y$ with the system gain $K$ as the conversion factor. Three noise sources are then added to the model with the following assumptions: the number of electrons $n_{e}$ is Poisson-distributed $\left(\sigma_{e}^{2}=\mu_{e}\right)$, the number of dark noise electrons $n_{d}$ is normally distributed $\left(\mu_{d} ; \sigma_{d}\right)$, and the quantization noise is uniformly distributed in $[-1 / 2,1 / 2]$, thus $\sigma_{q}^{2}=1 / 12$.

For the 4-step algorithm a simple relation between the recorded intensities $y_{i}$ and the estimated phase noise $\sigma_{\varphi \text {.mod }}$ can be derived based on this camera model [4]:

$\sigma_{\varphi \text { mod }}=\sqrt{\frac{\frac{K}{2}\left(y_{1}+y_{2}+y_{3}+y_{4}\right)+N_{4}}{\left(y_{1}-y_{3}\right)^{2}+\left(y_{2}-y_{4}\right)^{2}}}$

with the combined noise constant $N_{4}$ as:

$$
N_{4}=2 K^{2}\left(\sigma_{d}^{2}-\mu_{d}\right)+\frac{1}{6}
$$

In the following sections it will be shown how this general phase noise estimation can be applied to a given measurement system and further developed into a prediction method for the stochastic coordinate deviations of the measured surface points.

\section{Propagation into the object space}

The propagation of the stochastic phase deviations into the object space depends on the actual implementation of the measurement technique. In the example analyzed in this work the spatial coding of the surface via fringe projection is realized by a combination of phase-shifting and heterodyne evaluation. The whole image sequence consists of 12 images: three slightly different fringe widths with four $90^{\circ}$ phase shifts each. For each valid measurement point this yields a phase value $\varphi_{c, p, w}$ per camera index $c$, pixel index $p$ and fringe width index $w$ and in addition an estimated phase deviation $u_{c, p, w}$ according to eq. (4):

$u_{c, p, w}=\sigma_{\varphi \cdot \bmod }(c, p, w)$

For each pixel in both cameras the three independent phase measurements have to be normalized to a reference fringe width $\lambda_{\text {ref }}$ and averaged with the variances of the phase measurements as weighting factors. This results in the combined phase value $\varphi_{c, p}$ and the combined quality metric $u_{c, p}$ :

$$
\varphi_{c, p}=\frac{1}{\lambda_{r e f}} \frac{\sum_{w=1}^{3} \frac{\varphi_{c, p, w}}{\left(\lambda_{w} \cdot u_{c, p, w}\right)^{2}}}{\sum_{w=1}^{3} \frac{1}{\left(\lambda_{w} \cdot u_{c, p, w}\right)^{2}}}
$$

and

$$
u_{c, p}=\frac{1}{\lambda_{\text {ref }}} \sqrt{\frac{1}{\sum_{w=1}^{3} \frac{1}{\left(\lambda_{w} \cdot u_{c, p, w}\right)^{2}}}}
$$

For the triangulation of the object point coordinates an iterative method has been applied where the object point is found along a vector in $z$-direction by minimizing the phase differences $\delta \varphi_{1}$ and $\delta \varphi_{2}$ in both camera views. This process is depicted in fig. 3 .

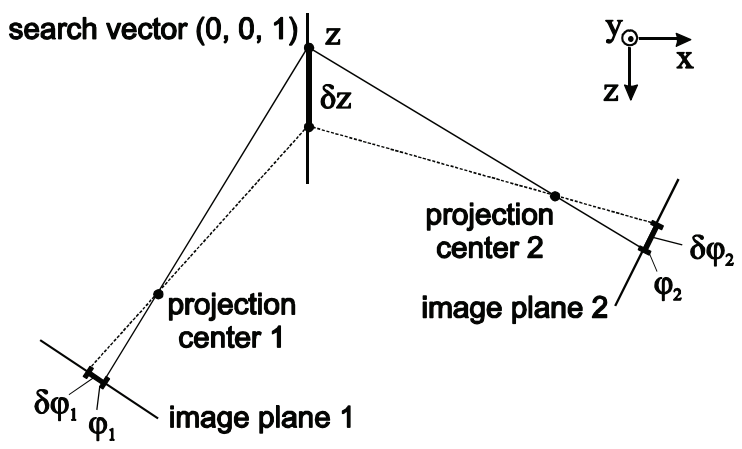

Fig. 3: Propagation of phase deviations into the object space in a fringe projection system with two cameras. The implementation is based on a passive triangulation along a search vector.

Because the image coordinates are calculated with subpixel resolution by means of a resection into the image plane, the corresponding phase value has to be interpolated. In the case of a bilinear interpolation that has been applied here, the interpolated phase value $\varphi_{c}$ can be calculated from the phase values $\varphi_{c, 1} \ldots \varphi_{c, 4}$ of the four surrounding pixels $p=1 \ldots 4$ by a weighted summation with the corresponding subareas $a_{c, p}$ as weighting factors:

$\varphi_{c}=\sum_{p=1}^{4} \varphi_{c, p} a_{c, p} \quad$ with $\sum_{p=1}^{4} a_{c, p}=1$

The influence of this bilinear interpolation on the quality metric can be incorporated as follows:

$u_{c}=\sqrt{\sum_{p=1}^{4}\left(\varphi_{c, p} a_{c, p}\right)^{2}}$ 
For the propagation of the estimated stochastic phase deviations $u_{1}$ and $u_{2}$ into the object space the evaluation principle depicted in fig. 3 results in additional coupling terms $C_{12}$ and $C_{21}$ between the sensitivities of both camera views, because for example $\delta \varphi_{1}$ is varying $\delta \varphi_{2}$ by a change of $z$ and vice versa:

$C_{12}=\frac{\left(\frac{\delta z}{\delta \varphi_{2}}\right)^{2}}{\left(\frac{\delta z}{\delta \varphi_{1}}\right)^{2}+\left(\frac{\delta z}{\delta \varphi_{2}}\right)^{2}}$

and

$$
C_{21}=\frac{\left(\frac{\delta z}{\delta \varphi_{1}}\right)^{2}}{\left(\frac{\delta z}{\delta \varphi_{1}}\right)^{2}+\left(\frac{\delta z}{\delta \varphi_{2}}\right)^{2}}
$$

With these coupling terms the resulting standard deviation of the estimated spatial noise $\sigma_{z \text { mod }}$ can finally be calculated as:

$$
\sigma_{z . \mathrm{mod}}=\sqrt{\left(u_{1} \frac{\delta z}{\delta \varphi_{1}} C_{12}\right)^{2}+\left(u_{2} \frac{\delta z}{\delta \varphi_{2}} C_{21}\right)^{2}}
$$

\section{Experimental validation of the noise model}

In addition to the object point $z$-coordinate itself, which is found by an iterative triangulation process, as described above, eq. (13) yields the corresponding estimated stochastic deviations $\sigma_{z \text { mod. }}$. The $\mathrm{x}$ - and y-coordinates compose a regular grid with a spacing of $20 \mu \mathrm{m}$ in the conducted experiments.

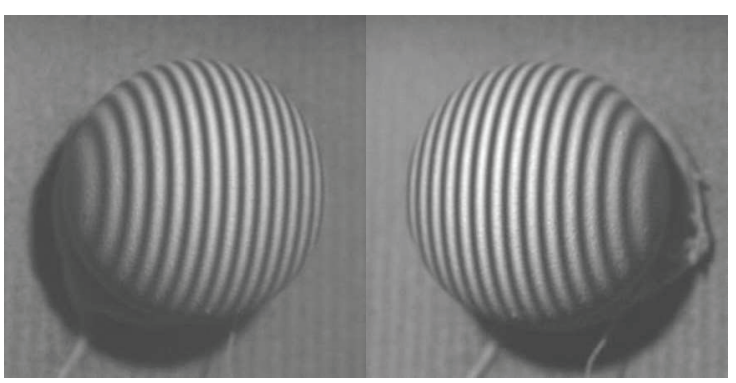

Fig. 4: Cropped camera views of the measured sphere with the fringe pattern projected onto it.

For the experimental validation a photogrammetric fringe projection system has been used, composed of two cameras with a resolution of $1024 \times 768$ pixel and a digital projector with a resolution of $800 \times 600$ pixel. The measure- ment volume is a cuboid with a size of about $20 \mathrm{~mm} \times 15 \mathrm{~mm} \times 10 \mathrm{~mm}$. As object under test a ball bearing sphere with a nominal diameter of $8 \mathrm{~mm}$ has been chosen. It has been prepared with an optically cooperative surface by spraying with titanium dioxide. In fig. 4 the views of both cameras onto the prepared sphere are shown. Because only the validity of the noise model is upon investigation, the geometric quality of the sphere, i.e. form deviations and roughness, is not relevant in these experiments. A sphere has been chosen because this leads to a good coverage of the $\beta$ and $\gamma$-parameter space in one single measurement. The variation of the surface normal from the pole of the sphere to the equator results in a decrease of $\beta$, whereas the large aperture and the focusing onto the pole leads to a small depth of focus and a decreasing $\gamma$ respectively. In fig. 5 the distribution of both parameters across the sphere has been visualized exemplarily for camera 1.
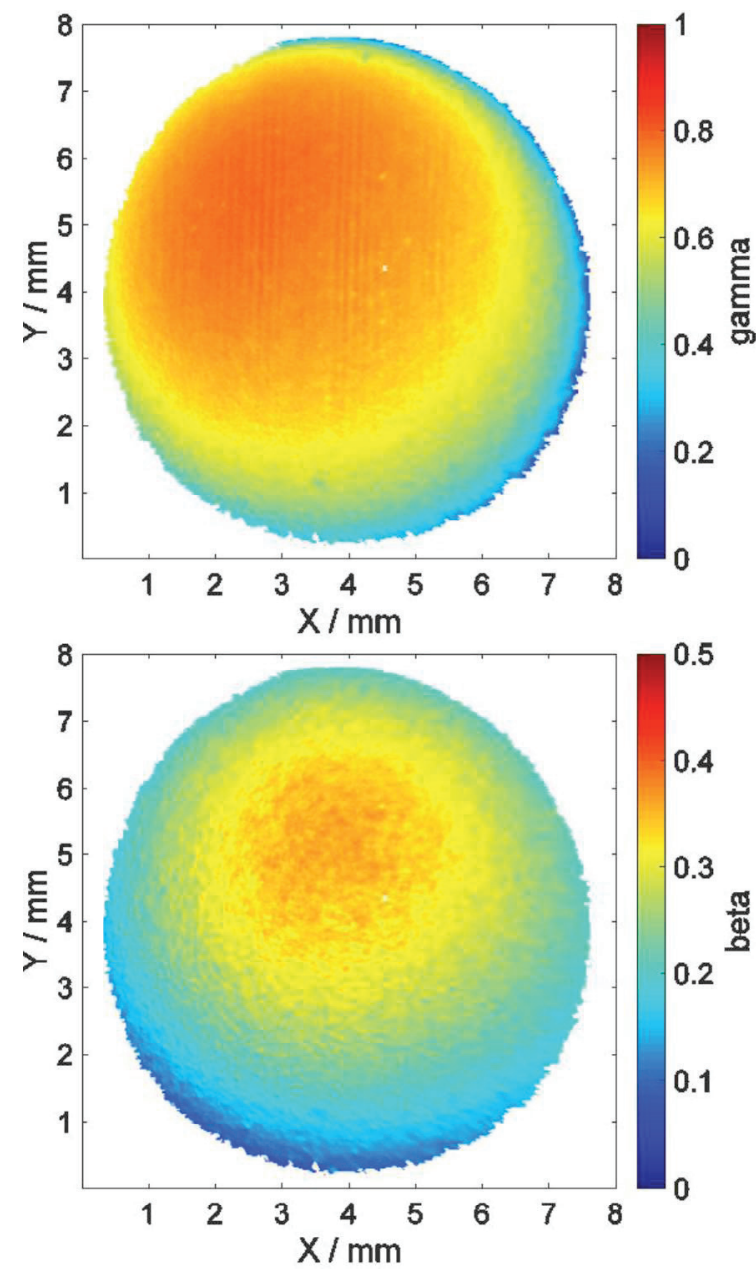

Fig.. 5: Visualization of the distribution of the parameters $\beta$ and $\gamma$ across the measured ball bearing sphere as recorded by camera 1. 
The resulting parameter space of combinations of $\beta$ and $\gamma$ that is available for the experimental validation of the noise model is shown in fig. 6 for both cameras. The difference between the distributions for each camera could be traced back to a slightly different aperture setting and a small off-center position of the sphere. The parameter space covered by these experiments can be considered as typical for a measurement of optically cooperative surfaces with a fringe projection system. A significantly different distribution is to be expected when the measurement object is made out of a volumescattering material for example. In this case the maximum visibility $\gamma_{\max }$ would be decreased, which in turn would typically lead to an increased illumination $\beta$ as the exposure time could be adapted accordingly.

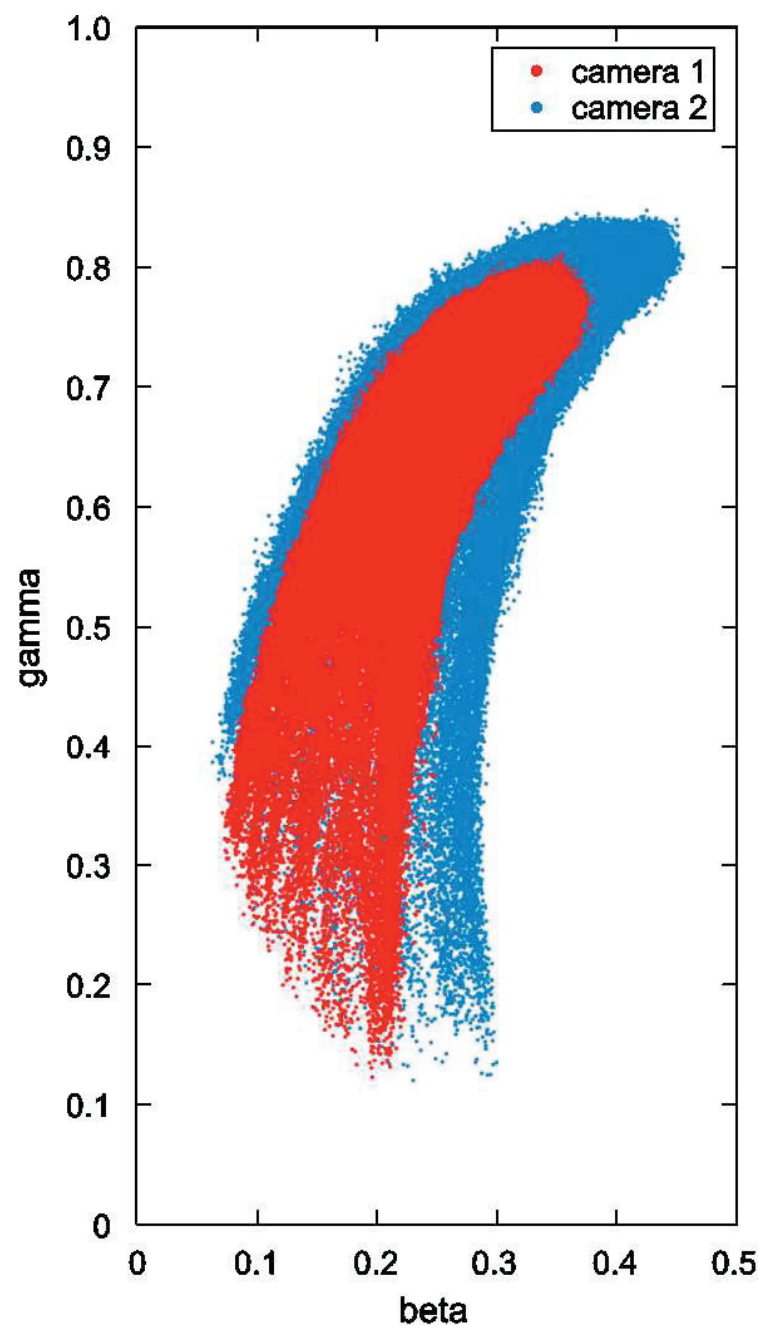

Fig. 6: Achieved coverage of the $\beta$ - and $\gamma$ parameter space of both cameras available for the experiments. This can be considered as typical for a fringe projection measurement of an optically cooperative surface.
The evaluation is based on a series of 800 repeated measurements of the sphere, lasting for about 2.5 hours. The subsequent analysis has been conducted only with measurement points that are valid across the whole measurement series, which has been the case for a total of 103.449 points.

It has been deducted from the data that thermal influences lead to a varying inner and outer orientation of the cameras over the course of the measurement series, resulting in an observed movement of the point cloud. For the centroid of the point cloud, a mean displacement in $z$-direction with a range of about $160 \mathrm{~nm}$ and a standard deviation of about $27 \mathrm{~nm}$ can be observed, which is not evenly distributed across the sphere. This influence superimposes the empirical data shown in the following sections and slightly affects the quality of the comparison between the empirically determined and the estimated spatial noise as the standard deviation for points with a high quality is about $1 \mu \mathrm{m}$.

From the whole series of 800 measurements the empirical standard deviation of the $z$ coordinate $\sigma_{z . \mathrm{emp}}$ has been calculated. The results are shown in fig. 7 (a). Points measured with good lighting conditions that are near the pole of the sphere feature a spatial noise in the order of $1 \mu \mathrm{m}$ whereas this value increases up to more than $5 \mu \mathrm{m}$ towards the boundary of the point cloud. For the validation this reference value has to be compared to the estimated spatial noise $\sigma_{z \text {.mod }}$ calculated from a single measurement of the series according to the noise model of eq. (13). The results are shown in fig. 7 (b) and it can be seen, that this is indeed consistent to fig. 7 (a). The very good agreement between empirical and estimated spatial noise can be better assessed in fig. 7 (c) where a row of the original data across the pole of the sphere is shown. It can be seen that both the low frequency variations and the high frequency interpolation effects are correctly estimated by the model.

The precision of the estimation method can be characterized by the distribution of $\sigma_{z \text { mod }}$ across all measurements of the series. The resulting distribution of the relative stochastic deviations of $\sigma_{z \text { mod }}$ exhibits a mean value of $3.61 \%$, a standard deviation of $1.52 \%$ and maximum values of about $10 \%$. It is strongly correlated with the expected spatial noise for the corresponding measurement point, with the points near the pole $\left(\sigma_{z} \approx 1 \mu \mathrm{m}\right)$ showing typical deviations of about $2 \%$ whereas in the boundaries of the point cloud $\left(\sigma_{z} \approx 5 \mu \mathrm{m}\right)$ this value increases to about $10 \%$. 
(a)

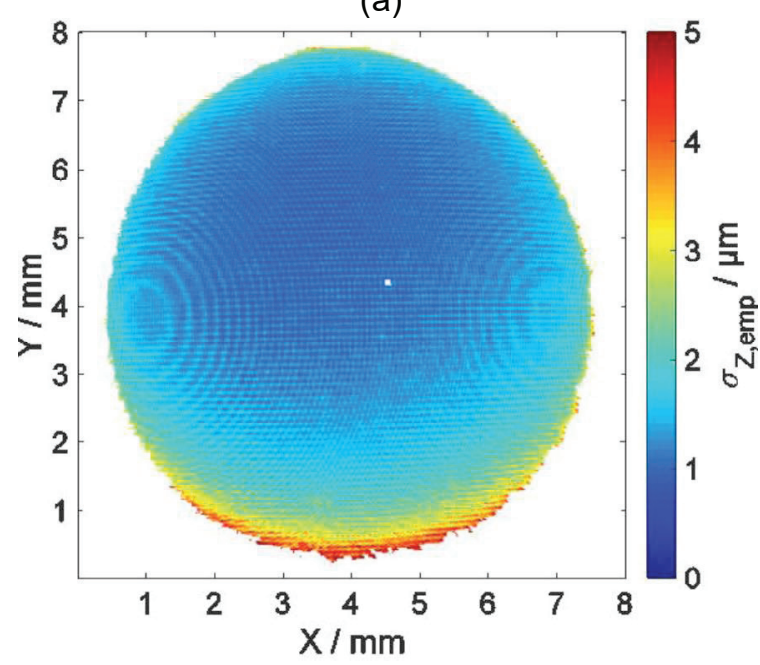

(b)

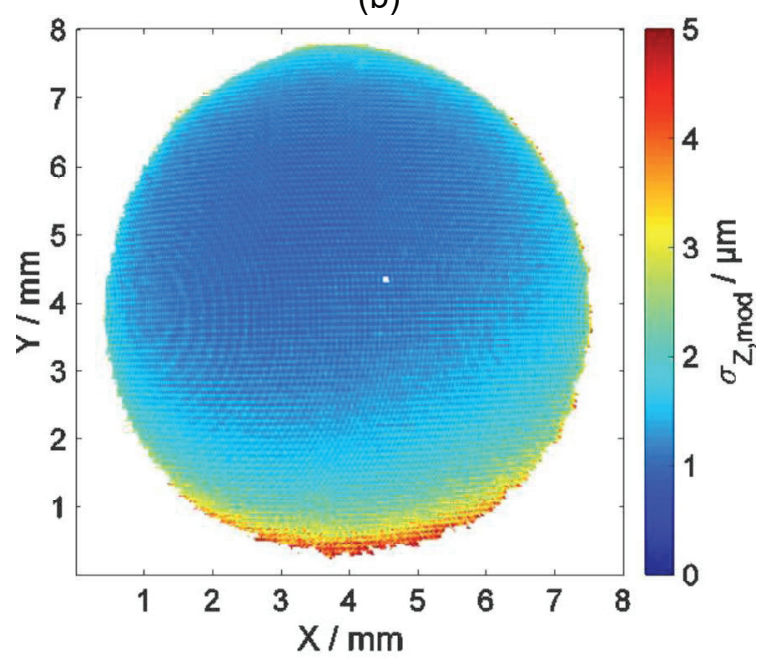

(c)

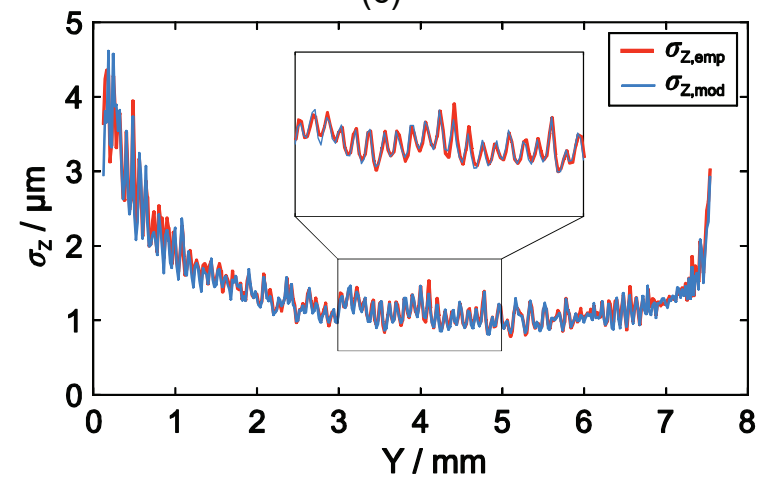

Fig. 7: Empirically determined reference data for the stochastic deviations of the measured $z$-coordinate $\sigma_{\text {z.emp }}$ (a) and the corresponding estimated value according to the noise model $\sigma_{z . m o d}(b)$. Both are highly consistent as can be seen in the plot of one single data row across the pole of the sphere (c).

\section{Summary}

In this work a model-based approach for the estimation of stochastic coordinate deviations, caused by camera noise in phase-measuring optical 3D measurement systems, has been proposed. In the case of a fringe projection system measuring an optically cooperative surface in a well-controlled environment this influence is the dominating factor. Thus the estimated spatial noise can directly be used as a point quality metric for example as a weighting factor. The precision of this estimation is better than $10 \%$ which can be seen as feasible for practical applications, especially given the fact that such information is generally not available at all for most industrial applications.

\section{Literaturnachweis}

[1] VDI/VDE 2634 Blatt 2:2012-8, Optische 3-DMesssysteme - Bildgebende Systeme mit flächenhafter Antastung

[2] VDA, Qualitätsmanagement in der Automobilindustrie, Band 5: Prüfprozesseignung, Eignung von Messsystemen, Mess- und Prüfprozessen, Erweiterte Messunsicherheit, Konformitätsbewertung, 2. vollständige überarbeitete Auflage 2010, aktualisiert 2011

[3] Surrel, Y.: Additive noise effect in digital phase detection. Applied Optics 36 (1997) 1, pp. 271276, doi: 10.1364/AO.36.000271

[4] Fischer, M.; Petz, M.; Tutsch, R.: Vorhersage des Phasenrauschens in optischen Messsystemen mit strukturierter Beleuchtung. In: tm Technisches Messen, Heft 79 (2012) 10, pp. 451-458, doi: 10.1524/teme.2012.0256

[5] European Machine Vision Association: EMVA Standard 1288 - Standard for Characterization of Image Sensors and Cameras, Release 3.0, November 29, 2010 